\title{
ACCURACY OF DIGITAL THREE-DIMENSIONAL ROOT POSITION PREDICTION VIA DIGITIZED MODELS
}

\author{
Mahmoud Abdullah El-Gendi*, Amr Emad El Dakroury** \\ Amr Ragab El Beialy*** and Mai Aboul Fotouh****
}

\begin{abstract}
Background: Accurate root position is integral for successful orthodontic treatment. Current methods of monitoring root position are either inaccurate, exhibit poor resolution, or use relatively large amount of radiation relative to the benefits for the patient. The purpose of this study was to present an approach that can monitor root position during orthodontic treatment with minimal radiation.
\end{abstract}

Methodology: At "Timepoint 0" a Cone-Beam Computed Tomography (CBCT) was taken for the patient before the start of the treatment. At "Timepoint 1" a CBCT was taken as a Reference Standard along with a digital extra-oral scan. A Predicted Root Position (PRP) assembly was made and compared to the Reference Standard to detect discrepancies between both.

Results: Mesiodistal tip analysis after indirect superimposition found displacement differences of $1.85 \pm 1.09 \mathrm{~mm}$ for the maxillary roots and $1.79 \mathrm{~mm} \pm 1.16 \mathrm{~mm}$ for the mandibular roots. For buccolingual torque, displacement differences of $2.13 \pm 1.52 \mathrm{~mm}$ for the maxillary roots and 2.29 $\mathrm{mm} \pm 1.59 \mathrm{~mm}$ for the mandibular roots. For root tip displacement, displacement differences of $1.57 \pm 0.86 \mathrm{~mm}$ for the maxillary roots and $1.44 \mathrm{~mm} \pm 0.82 \mathrm{~mm}$ for the mandibular roots.

Conclusions: This method was found to have the potential to accurately predict the root position during treatment in three dimensions.

\section{INTRODUCTION}

The demand on orthodontic treatment has witnessed a significant increase over the years. ${ }^{1,2}$ And one of the paramount objectives of a successful orthodontic treatment is to achieve root parallelism. ${ }^{3}$ So, in order to be able to visualize the root positions, a panoramic radiograph is to be taken near the end of the finishing stage just before the removal of the fixed appliance to make sure roots are in the positions they are intended to be, and to make any fine tuning if needed. In addition, we may sometimes need to visualize root positions along the course of the treatment. But according to ALARA (As Low

*Orthodontics Master Cairo University.

**Professor of Orthodontics, Orthodontic Department, Faculty of Dentistry, Cairo University.

****Associate Professor of Orthodontics, Orthodontic Department, Faculty of Dentistry, Cairo University.

**** Lecturer of Orthodontics, Orthodontic Department, Faculty of Dentistry, Cairo University 
As Reasonably Achievable) principle, the patient should be exposed to the least amount of diagnostic necessary radiation to avoid the possible harmful effects. However, the drawbacks of panoramic imaging and its inherent limitations significantly diminish the efficiency of accurately visualizing the exact root positions of teeth. Therefore, sometimes a more precise Cone-Beam Computed Tomography (CBCT) image is to be taken to precisely locate root positions 3-dimensionally ${ }^{4}$, to make necessary adjustments and detailing of the occlusion to reach satisfactory results for both parties -the clinician and most importantly the patient. Unfortunately, a drawback in taking a CBCT is its relatively high radiation dose, which according to a cost/benefit analysis can shift the clinician to spare the patient this procedure and resort to a less quality image but that will save the patient this difference in dosage. ${ }^{5}$ As we are now in the era of digitization, dentistry and the field of orthodontics in specific is no exception. So, the perpetual advances that keep on occurring in this field seem to be very promising. An example is the evolution of three-dimensional scanners in both the extra-oral and intra-oral forms. ${ }^{6,7}$. So taking advantage of these technologies to aid in providing orthodontic treatment seekers more precise, easier, faster and less troublesome solutions becomes a reasonable need. ${ }^{8}$.

So, in light of the previous, a study was done to predict root positions via digitized stone models and CBCTs. ${ }^{9}$ But, this was a study that was done on a single patient retrospectively which is unreliable to provide a guide for its use. In addition to this, it was done using post-treatment scan models which does not guarantee accuracy of the scan if root positions are to be visualized mid-treatment while the fixed appliance is still in place. This is what provoked this` current study in an attempt to prove this tool reliable.
Enough for use in prediction of root position along the course of active orthodontic treatment. In this tool, individual teeth are to be segmented pre-operatively from CBCTs. Cast models will be digitized and exported in "STL" format to do superimposition between the Predicted Root Position (PRP) and actual positions in the pre-finishing stage.

\section{MATERIAL \& METHODS}

This prospective study was approved by the Research Ethics Committee of the Faculty of Dentistry, Cairo University. Patient selection for this trial was done in the outpatient clinic of the Department of Orthodontics, Faculty of Dentistry, Cairo University after clinical and radiographic examination proved them eligible for a nonextraction based orthodontic treatment. Eligible patients were enrolled in a consecutive series. Non-syndromic, Class I molar \& canine, nonextraction $2-4 \mathrm{~mm}$ crowding and spacing cases were included. All included patients completed their orthodontic treatment by the same operator. The sample of this trial included 4 male and 6 female patients comprising 100 maxillary teeth and 98 mandibular teeth due to the absence of mandibular second premolars in one patient. For every patient, Maxillo-Mandibular CBCT, an intra-oral and extraoral photographs and study casts were taken at "Timepoint 0". Afterwards, all patients underwent routine orthodontic treatment ${ }^{*}$ going through the following wire sequence. $0.014 \mathrm{NiTi}-0.016 \mathrm{NiTi}$ - 0.016 Stainless Steel -0.016 x 0.022 Stainless Steel. Using Ondemand3D ${ }^{* *}$ software, individual teeth segmentation was done pre-operatively using the CBCT at "Timepoint 0" as follows: The "Bone Threshold" was chosen and the threshold slider was manually adjusted so as to achieve maximum root visualization. The "Draw Mask" tool from the

\footnotetext{
* American Orthodontic Bracket system, Roth Prescription, 0.018-inch slot size

** Cybermed Inc., Republic of Korea
} 
segmentation section in the left toolbar was chosen to delineate the outline of the tooth to be segmented, selecting the tooth material and "Select as a new object" was chosen, then tooth segmentation from all possible views by delineating the precise outline of the tooth was done and then what was outside of the tooth's margins was removed using the "Remove the region" option, alternating between "Bone" and "Teeth" threshold for better visualization and optimum results as seen in (Fig.1)

After finishing this step, the tooth was renamed from the lower left "Fine Tuning" section and exported as an STL file. The previous steps were repeated for all upper and lower permanent teeth.

After traditional orthodontic fixed appliance therapy was started and at the pre-finishing stage, another CBCT was obtained. At the same time, an alginate impression was obtained for both dental arches using Regular set CA 37, poured within 15 minutes using high strength dental stone type IV and scanned extra-orally using inEos X5 extra-oral scanner. Teeth were segmented at this timepoint and exported as one STL file to act the reference

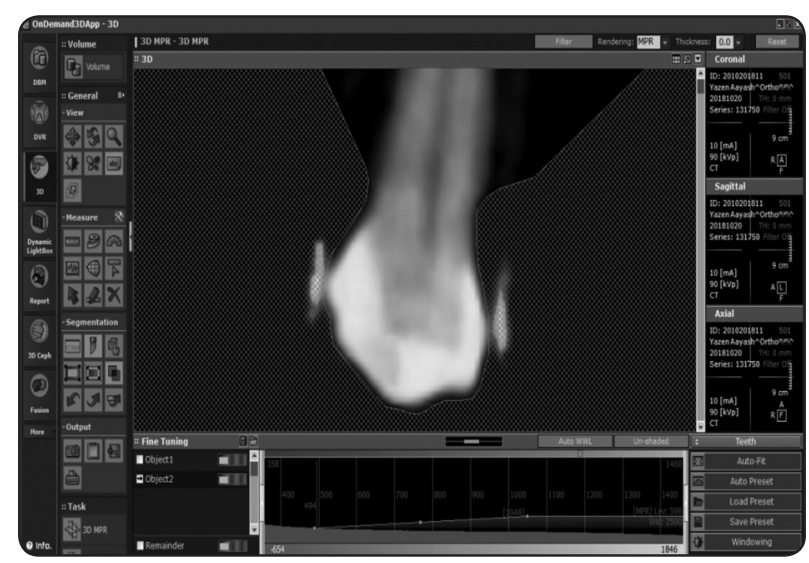

Fig. (1): OnDemand3D window showing segmentation of the crown

\footnotetext{
* Cybermed Inc., Republic of Korea
}

standard. The separately segmented teeth were then superimposed on the digital model using "N-point Registration", "Interactive Rotate" and "Interactive Translate" tools to obtain the root position prediction (Index Test) using 3-matic Materialise* software. Now both the index test and the reference standard were superimposed over each other using " $\mathrm{N}$ - point Registration" tool and a color map analysis was done to ensure the precision of the superimposition before the start of the measurement analysis. Fine tuning of the automatic superimposition was further done using the "Interactive rotation and translation" functions. Difference in mesiodistal tip was measured for each tooth from the labial aspect and the buccolingual torque from the proximal aspect using the "Measure Angle" tool by drawing a long axis for both teeth (the Index and the reference standard) and measuring the angle in between. Root Tip Displacement was measured between the prediction and the reference standard by measuring the linear distance between the root apices using the "Measure Distance" tool as shown in (Fig. 2).

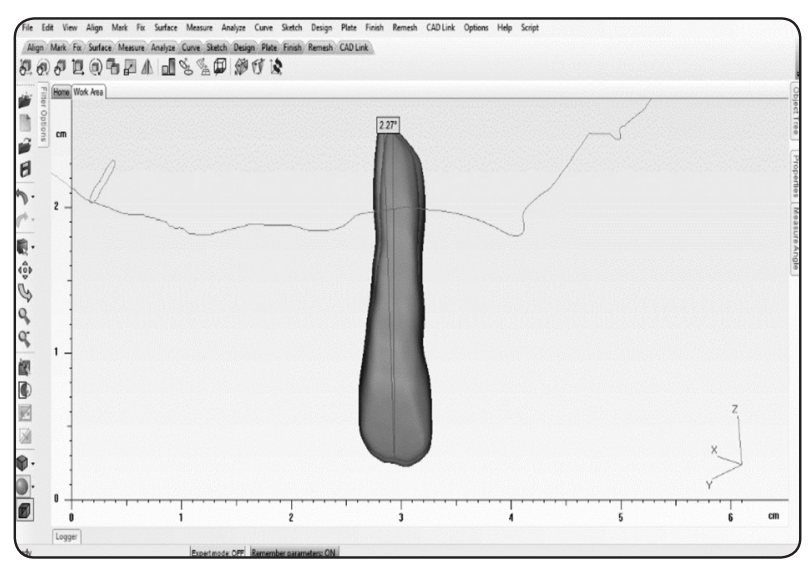

Fig. (2): Window of 3-matic Software showing the predicted tooth slightly transparent to measure the difference in tip between the prediction and reality by drawing a long axis of both teeth and measuring the angle in between using "Measure Angle" tool 


\section{RESULTS}

The results of this trial are presented under the following titles: Intra-Observer Reliability (Table $1)$, Mesiodistal displacement ( $\Delta$ Tip) (Table 2). The collected data were obtained through case control trial performed using digitized models on selected patients according to selected eligibility criteria.

Data were statistically analyzed by Microsoft Excel ${ }^{\circledR} 2016 *$, Statistical Package for Social Science (SPSS) ${ }^{\circledR}$ Ver. $24^{* *}$. and Minitab ${ }^{* * *}{ }^{\circledR}$ statistical software Ver. 16.

TABLE (1) Intra-observer reliability for all teeth

\begin{tabular}{|c|c|c|c|c|c|c|c|c|}
\hline \multicolumn{9}{|c|}{ All Teeth } \\
\hline & \multicolumn{2}{|c|}{ Trial I } & \multicolumn{2}{|c|}{ Trial II } & \multirow[b]{2}{*}{ P-value } & \multirow{2}{*}{$\begin{array}{c}\text { Cronbach`s } \\
\text { Alpha }\end{array}$} & \multirow{2}{*}{$\begin{array}{l}\text { Intraclass } \\
\text { Correlation } \\
\text { Coefficient }\end{array}$} & \multirow[b]{2}{*}{ Reliability } \\
\hline & $\begin{array}{l}\text { Mean } \\
(\mathrm{mm})\end{array}$ & $\pm \mathrm{SD}$ & $\begin{array}{l}\text { Mean } \\
(\mathrm{mm})\end{array}$ & $\pm \mathrm{SD}$ & & & & \\
\hline$\Delta$ Tip & 1.82 & 1.125 & 1.7 & 1.14 & 0.45 & 0.754 & 0.695 & High \\
\hline$\Delta$ Torque & 2.21 & 1.55 & 2.20 & 1.59 & 0.46 & 0.745 & 0.655 & High \\
\hline $\begin{array}{c}\text { Root Tip } \\
\text { Displacement }\end{array}$ & 1.57 & 0.86 & 1.4370 & .68862 & .555 & 0.722 & 0.623 & High \\
\hline
\end{tabular}

TABLE (2): Accuracy of Root Position Prediction (Tip, Torque, Root Tip Displacement)

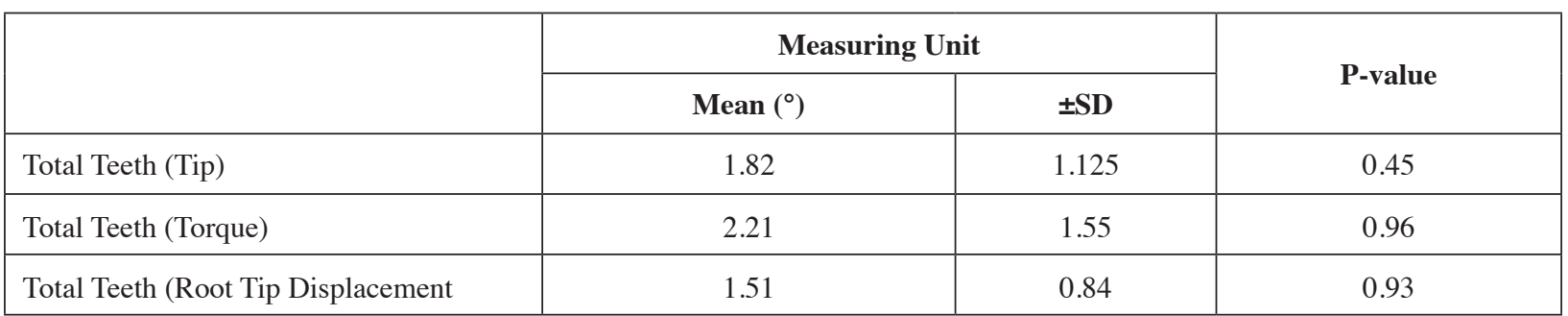

\section{DISCUSSION}

The final root position after orthodontic treatment influences the periodontal health, restoration placement and occlusion. Also, according to the $\mathrm{ABO}$ (American Board of Orthodontics) ${ }^{1}$, it is necessary to assess root angulations at the end of the treatment to evaluate the Cast-Radiograph Evaluation (CRE) to render a case "well-treated", and points are applied where roots are not aligned properly or if they touch each other.
However, several previous research articles ${ }^{2,3,4}$ have denounced that using such a modality is not accurate. Even in the CRE, canines are not considered due to major distortion that occurs at that region on the radiograph. Furthermore, it is advised, according to ALARA (As Low As Reasonably Achievable) principle, to decrease the amount of radiation as low as possible. So, in light of the previous, it was consequential to contrive an innovative technique to visualize the root position accurately. And at the same time, eliminates the

\footnotetext{
* Microsoft Cooperation, USA.

** IBM Product, USA.

*** Minitab LLC, USA.
} 
necessity to obtain a pre-finishing, less accurate panoramic radiograph. A tool that can be an ideal resort in case of orthodontic malocclusions that necessitate a CBCT before the start of the treatment.

The first trial ${ }^{5}$ to attempt such a technique was prompted using an ex-vivo typodont where the CBCT was sent to a third party company and it sent the STL files of teeth separately afterwards.

Another retrospective study ${ }^{5}$ re-attempted the technique using the records of one patient incorporating the "Anatomodel" modeling service. Then, a prospective study ${ }^{6}$ was done three years later using records taken at a dedicated reset appointment. However, in this trial a manual segmentation technique was done to eliminate the need for a third party.

For the sake of this trial, a maxillo-mandibular CBCT image was taken before the start of the orthodontic treatment and teeth were manually segmented and kept aside as STL files.

It should be noted that the process of root position prediction using this methodology was time-consuming and subjected to several variables starting from the quality of the CBCT, the accuracy of the software used and its efficiency. And finally, the clinician's personal experience.

It should be taken into consideration that segmenting the occlusal anatomy was quite challenging to rotate the tooth from every possible aspect to make sure it was totally segmented from the surrounding bone.

To assess the accuracy of the root position, it was compared against the pre-finishing CBCT segmented teeth acting as the reference standard for research purposes only. In a clinical setting, the need for panoramic or CBCT imaging at the prefinishing stage may be eliminated.

To detect the accuracy of superimposition before starting to compare between the difference in tip, torque and root tip displacement, the laser-scan cast was removed from the assembly and a color map analysis was done between the root position prediction and the reference standard.

A similar technique was demonstrated in just one in-vivo case. ${ }^{7}$ Another trial was made on an ex vivo typodont. ${ }^{5}$ But this trial was the first to use a manual segmentation technique to separate teeth from the surrounding bone and was done on multiple patients in-vivo.

\section{CONCLUSIONS}

From the results of the clinical and statistical analyses, and within the limitations of this trial, the following conclusions could be withdrawn. This technique for root position prediction proved to be an accurate prediction tool for the root position regarding mesiodistal tip, buccolingual torque and root tip displacement. This technique is extremely technique-sensitive, time consuming and subject to the human factor in both teeth segmentation and superimposition. This tool was able to predict the root position accurately within the clinically accepted premises regarding the mesiodistal displacement. This technique could be considered as a valid, reliable tool for root position prediction.

\section{REFERENCES}

1. Casko JS, Kokich VG, Cangialosi TJ. Objective grading system for dental casts and panoramic radiographs AMERICAN BOARD OF ORTHODONTICS. 1996: 589-599.

2. Mckee IW, Glover KE, Williamson PC, Lam EW, Heo G, Major PW. The Effect of Vertical and Horizontal Head Positioning in Panoramic Radiography on Mesiodistal Tooth Angulations. Angle Orthod. 2001;71(6):442-451.

3. Garcia-Figueroa MA, Raboud DW, Lam EW, Heo G, Major PW. Effect of buccolingual root angulation on the mesiodistal angulation shown on panoramic radiographs. Am J Orthod Dentofac Orthop. 2008;134(1):93-99. doi:10.1016/j.ajodo.2006.07.034

4. Owens AM, Johal A. Near-end of treatment panoramic radiograph in the assessment of mesiodistal root angulation. Angle Orthod. 2008;78(3):475-481. doi:10.2319/040107161.1 
5. Lee RJ, Pham J, Choy M, et al. Monitoring of typodont root movement via crown superimposition of single conebeam computed tomography and consecutive intraoral scans. Am J Orthod Dentofac Orthop. 2015;145(3):399409. doi:10.1016/j.ajodo.2013.12.011

6. Lee RJ, Pi S, Park J, Nelson G, Hatcher D, Oberoi S. Three-dimensional evaluation of root position at the reset appointment without radiographs: a proof-of-concept study. Prog Orthod. 2018;19(1). doi:10.1186/s40510-0180214-4

7. Lee RJ, Weissheimer A, Pham J, et al. Three-dimensional monitoring of root movement during orthodontic treatment. Am J Orthod Dentofac Orthop. 2015;147(1):132-142. doi:10.1016/j.ajodo.2014.10.010 